Research Paper

\title{
Increased Locomotor Activity and Non-Selective At- tention and Impaired Learning Ability in SD Rats after Lentiviral Vector-Mediated RNA Interference of Homer Ia in the Brain
}

\author{
Qin Hong ${ }^{* 1}$, Lei Yang*2, Min Zhang1, Xiao-Qin Pan², Mei Guo², Li Fei², Mei-Ling Tong 1,2, Rong-Hua Chen², \\ Xi-Rong Guo ${ }^{1,2, \square}$, Xia Chi ${ }^{1,2,}$, \\ 1. State Key Laboratory of Reproductive Medicine, Department of Pediatrics, Nanjing Maternity and Child Health Hospital of Nanjing \\ Medical University, Nanjing 210004, China; \\ 2. Institute of Pediatrics of Nanjing Medical University, Nanjing 210029, China. \\ * The authors Qin Hong and Lei Yang contributed equally to this work.
}

\begin{abstract}
$\triangle$ Corresponding author: Dr Xia Chi and Dr Xi-Rong Guo, State Key Laboratory of Reproductive Medicine, Department of Pediatrics, Nanjing Maternity and Child Health Care Hospital Affiliated to Nanjing Medical University, Nanjing 210029, China; Institute of Pediatrics of Nanjing Medical University, Nanjing 210029, China, Tel.: 86-25-8222-6476 (Xia Chi), 86-25-8222-2996 (Xi-Rong Guo) Fax: 86-25-8446-0507 E-mail: chixia2001@njmu.edu.cn (Xia Chi), xrguo@njmu.edu.cn (Xi-Rong Guo).
\end{abstract}

(C) Ivyspring International Publisher. This is an open-access article distributed under the terms of the Creative Commons License (http://creativecommons.org/ licenses/by-nc-nd/3.0/). Reproduction is permitted for personal, noncommercial use, provided that the article is in whole, unmodified, and properly cited.

Received: 2012.07.17; Accepted: 2012.12.24; Published: 2012.12.27

\begin{abstract}
Our previous studies found that Homer Ia, a scaffolding protein localized at the post-synaptic density (PSD) of glutamatergic excitatory synapses, is significantly down-regulated in the brain of spontaneous hypertensive rats (SHR), an animal model of attention deficit hyperactivity disorder (ADHD). Furthermore, a first-line treatment drug for ADHD, methylphenidate, can up-regulate the expression of Homer la. To investigate the possible role of Homer la in the etiology and pathogenesis of ADHD, a lentiviral vector containing miRNA specific for Homer la was constructed in this study. Intracerebroventricular injection of this vector into the brain of Sprague Dawley (SD) rats significantly decreased Homer I a mRNA and protein expression levels. Compared to their negative controls, these rats displayed a range of abnormal behaviors, including increased locomotor activity and non-selective attention and impaired learning ability. Our results indicated that Homer la down-regulation results in deficits in control over behavioral output and learning similar to ADHD.
\end{abstract}

Key words: Homer 1a; RNAi; lentiviral vector; ADHD; behavior.

\section{Introduction}

Attention deficit hyperactivity disorder (ADHD, MIM143465) is a pervasive neurobehavioral disorder affecting approximately $5 \%$ of children and adolescents and $3 \%$ of adults [1]. Children with ADHD are characterized by two or three core clusters of symptoms-impaired sustained attention and hyperactivity/impulsiveness that develop gradually in familiar situations, with impairment manifesting before the age of seven. Despite ADHD possibly being the most studied condition in child psychiatry worldwide, the exact causes and mechanisms underlying this disorder are not yet completely understood. Fortunately, animal models combined with modern molecular biology techniques provide powerful tools for investigating the pathogenesis of ADHD.

Our previous studies found that Homer 1a, a 
scaffolding protein localized at the postsynaptic density (PSD) of glutamatergic excitatory synapses, is expressed at a significantly lower level in the brain of spontaneous hypertensive rats (SHR), an ADHD animal model, compared with control Wistar-Kyoto (WKY) rats [2-4]. Meanwhile, exposure to methylphenidate (MPH), one of the most commonly prescribed psychoactive stimulants for the treatment of ADHD, has been found to up-regulate the expression of Homer 1a $[3,4]$. Therefore, the regulation of Homer 1a may be closely related to ADHD. Homer 1a is the short form of the Homer protein, which is functionally different from the long form due to lack of the C-terminal coiled-coil structure and leucine zipper motifs. Homer 1a competes with the long-form Homer proteins to combine with some PSD related components, such as metabotropic glutamate receptors (mGluR1, mGluR5), inositol trisphosphate receptors (IP3Rs) and Shank, thus behaving as a dominant negative in PSD remodeling and may represent a mechanism of synaptic plasticity and a putative target for both pharmacotherapy and pharmacogenomics of behavioral disorders [5]. Converging preclinical observations have indicated a potential role of Homer 1a in behavioral pathologies associated with neuropsychiatric disorders, such as addiction and/or alcoholism, depression, anxiety, epilepsy and schizophrenia [6]. Homer 1 knockout mice exhibit pronounced learning deficits during acquisition in both the Morris water maze and radial arm maze tests, indicating poor reference and working memory [7,8]. Meanwhile, Homer 1a overexpression can improve cognitive function in rats [9]. Environmental enrichment reverses the impaired exploratory behavior of rats with early life seizures and increases Homer 1a expression in the hippocampus [10]. Synaptic long-term potentiation is an acknowledged cellular model of learning and memory in the hippocampus which can also induce Homer 1a expression [11]. Therefore Homer $1 \mathrm{a}$ is a critical gene for regulating behavior. Accordingly, we speculate that Homer 1a may contribute to the etiology and development of ADHD.

RNA interference (RNAi) has recently emerged as a useful genetic tool for silencing gene expression by triggering post-transcriptional degradation of homologous transcripts through a multi-step mechanism involving double-stranded small silencing RNA [12]. Several types of small silencing RNAs have now been discovered, including small interfering RNAs (siRNAs), microRNAs (miRNAs) and PIWI-interacting RNAs (piRNAs) [13]. As ADHD is a behavior disorder related to brain development, the primary technique used in this study was in vivo delivery of an RNAi vector to neurons. Traditional direct transfection of siRNAs achieves only a transient reduction in target gene expression in cell culture models [14]. In order to improve the efficiency of RNAi, we designed artificial miRNAs with $100 \%$ homology to our target sequence, which can more efficiently cleave the target than traditional siRNA [15]. We also chose a lentiviral vector, which is an efficient transducer for a wide variety of primary cells, whether proliferating or quiescent, to achieve long-term cell modification in vivo [16]. Lentiviral vector can efficiently integrate the objective gene into the host cell genome, and is divided with the splitting of the cell's genome. In addition, it can infect and integrate into non-dividing cells. Thus, lentvirus vector-mediated expression of RNAi can achieve effective, stable gene silencing in biological systems. Therefore, in this study a lentiviral vector containing an artificial miRNA specific for Homer 1a was constructed and delivered into the rat brain via intracerebroventricular injection, which resulted in seven days silencing of Homer 1a. The behavioral phenotype of these rats and expression of Homer 1a in the brain was observed in order to confirm our previous assumptions that Homer 1a may be related to ADHD.

\section{Materials and methods}

This study involved three steps experimental stages: (1) Construction of a lentiviral vector containing an artificial miRNA specific for Homer 1a; (2) In vitro validation: The primary neural cells obtained from the Sprague Dawley (SD) rat's cortices was cultured for two days and then transduced with the lentiviral vector containing Homer 1a-specific miRNA (Homer 1a-RNAi-LV) or negative control (NC-LV) at the appropriate multiplicity of infection (MOI) determined pre-experimentally. The expression levels of Homer 1a mRNA and protein were then evaluated using quantitative real-time RT-PCR (qPCR) and Western blot. (3) In vivo validation: $24 \mathrm{SD}$ rats were divided into two groups of 12 in each and given Homer 1a-RNAi-LV (RNAi group) or NC-LV (NC group) by intracerebroventricular injection to examine the effects of Homer 1a RNAi on behaviors in the Làt maze and the Morris water maze tests. After completing the behavioral assessments, we randomly selected four rats from each of the two groups. The mRNA and protein expression level of Homer 1a was detected by qPCR and Western blot, respectively.

\section{Construction of a lentiviral vector containing an artificial Homer Ia-specific miRNA}

Four pairs of artificial single-stranded DNA oligonucleotides were designed and synthesized: one in each pair encoding the target pre-miRNA ("forward 
strand" oligo) according to the Homer 1a reference sequence (Genbank accession no. AB003726.1), and the other was its complement ("reverse strand" oligo). After annealing each pair of oligos, the four double chain miRNAs were inserted into the miRNA expression vector $\mathrm{pcDNA}^{\mathrm{TM}} 6.2-\mathrm{GW} / \mathrm{miR}$ from the BLOCK-iT ${ }^{\mathrm{TM}}$ Pol II miR RNAi Expression Vector Kit (Invitrogen, Carlsbad, CA, USA). The four miRNA expression plasmids were transformed into Escherichia coli $\mathrm{DH} 5 \alpha$ and confirmed by sequencing. The four miRNA expression vectors and negative control vector were then transfected into rat $\mathrm{H} 9 \mathrm{c} 2$ cells separately to evaluate their RNAi efficiencies by qPCR. Homer1a-1 miRNA induced the greatest down-regulation of about $50 \%$ (see Figure 1). Thereafter, the rat Homer 1a-1 miRNA expression cassette vector and viral packaging system (Invitrogen, containing an optimized mixture of the two packaging plasmids) were co-transfected into 293 cells to generate competent lentiviruses, designated as Homer 1a-RNAi-LV. The viral supernatant was harvested 48 $\mathrm{h}$ after transfection and filtered through a $0.45-\mathrm{mm}$ cellulose acetate filter and frozen at $-80^{\circ} \mathrm{C}$. The average titer was $3.38 \times 10^{7}$ infectious units (ifu) $/ \mathrm{mL}$. The negative miRNA plasmid was also packaged and used as a negative control, designated as NC-LV. The $\mathrm{NC}-\mathrm{LV}$ is generally applicable of the control, and is particular for this vector. This control theoretically has no effect on any gene; therefore it was used as a negative control. The sequence is $5^{\prime}$-TGCTGAAATG TACTGCGCGTGGAGACGTTTTGGCCACTGACTG ACGTCTCCACGCAGTACATTT-3'.

A
\begin{tabular}{|l|l|}
\hline Homerla-1-F & 5'-TGCTGCACAGTAACTGCATGCTTGCTGTTTTGGCCACTGACTGACAGCAAGCACAGTTACTGTG -3' \\
\hline Homer1a-1-R & 5'-CCTGCACAGTAACTGTGCTTGCTGTCAGTCAGTGGCCAAAACAGCAAGCATGCAGTTACTGTGC-3' \\
\hline Homer1a-2-F & 5'-TGCTGATTCTGAGCTGGCTCAGCCCTGTTTTGGCCACTGACTGACAGGGCTGACAGCTCAGAAT -3' \\
\hline Homerla-2-R & 5'-CCTGATTCTGAGCTGTCAGCCCTGTCAGTCAGTGGCCAAAACAGGGCTGAGCCAGCTCAGAATC-3' \\
\hline Homerla-3-F & 5'- TGCTGTCCTCCTGCTGATTCCTGTGAGTTTTGGCCACTGACTGACTCACAGGACAGCAGGAGGA-3' \\
\hline Homer1a-3-R & 5'-CCTGTCCTCCTGCTGTCCTGTGAGTCAGTCAGTGGCCAAAACTCACAGGAATCAGCAGGAGGAC-3' \\
\hline Homerla-4-F & 5'- TGCTGAATCCCAGTCCATAAACAGTGGTTTTGGCCACTGACTGACCACTGTTTGGACTGGGATT-3' \\
\hline Homerla-4-R & 5'-CCTGAATCCCAGTCCAAACAGTGGTCAGTCAGTGGCCAAAACCACTGTTTATGGACTGGGATTC-3' \\
\hline
\end{tabular}

B

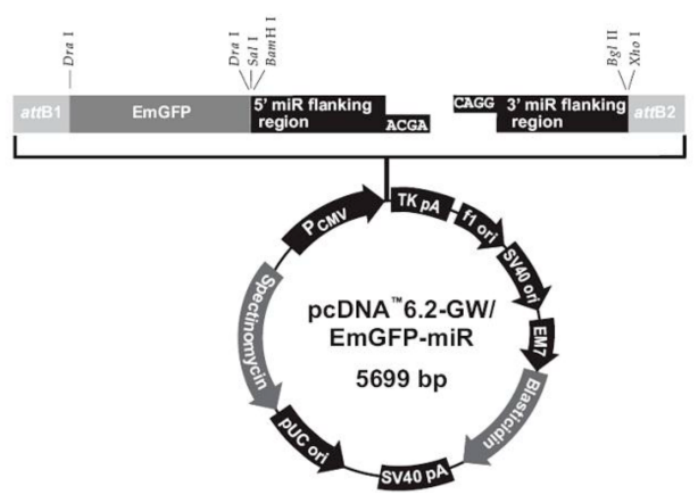

$\mathrm{C}$

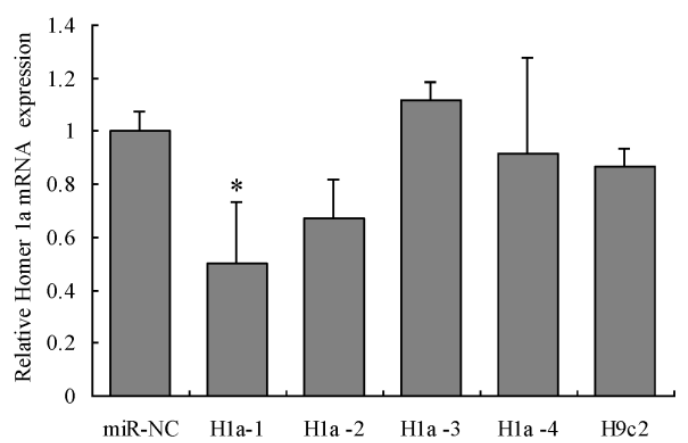

Figure I. Design of Homer la-specific miRNA. (A) Sequences of four pairs of artificial Homer la-specific miRNA oligos. (B) miRNA expression vector map. (C) Homer la-I miRNA was the most effective sequence among the four candidates. $(F=3.94, P<0.05)$. miR-NC indicates cells transfected with negative control miRNA. Hla-I, $\mathrm{HIa}-2, \mathrm{HIa}-3$ and $\mathrm{HIa}-4$ indicate cells transfected with Homer la-I, Homer Ia-2, Homer Ia-3, Homer Ia-4 miRNA, respectively. H9c2 indicates cells without transfection. 


\section{Intracerebroventricular injection of lentivi- ruses}

24 rats (aged 4 weeks) were obtained from the Experimental Animal Center of Nanjing Medical University. The animal holding room was illuminated on a 14-h/10-h light/dark cycle, and the temperature was maintained at $22-24^{\circ} \mathrm{C}$. Water and food were available ad libitum. The animals were allowed 1 week to acclimate to their new surroundings before the experiments began. The experimental procedures followed the guidelines for animal care and experiments of Nanjing Medical University. Weights of the rats were measured weekly, ranged from 70-80g (4 weeks old) at the beginning to $210-230 \mathrm{~g}$ (7 weeks old) at the end (see body weight curve in figure 2). Rats were anesthetized using 20\% urethane $(1 \mathrm{ml} / 100 \mathrm{~g})$ and placed in a stereotaxic apparatus. The skull was exposed by a midline incision, and a hole was drilled in the appropriate location using specific coordinates $(($ anterioposterior $(\mathrm{AP})=-1.2 \mathrm{~mm}$; mediolateral $(\mathrm{ML})$ $=1.3 \mathrm{~mm}$; and dorsoventral $(\mathrm{DV})=-3 \mathrm{~mm})$ with respect to the Bregma) [17]. The concentrated virus was injected into the lateral ventricles $(10 \mu \mathrm{l}$ per side for each rat, at the rate of $1 \mu \mathrm{l} / \mathrm{min})$. The microinjector was removed 5 min later, and then the scalp was sutured and disinfected. The rats were kept warm until recovery.

Table 2. Body weight curve of rats after intracerebroventricular injection of Homer la-RNAi-LV (RNAi) or negative control NC-LV (NC).

\begin{tabular}{lllll}
\hline & 4 weeks & 5 weeks & 6 weeks & 7 weeks \\
\hline NC (g) & $77.08 \pm 8.20$ & $149.67 \pm 19.54$ & $215.00 \pm 20.16$ & $226.17 \pm 19.10$ \\
RNAi (g) & $76.58 \pm 5.09$ & $145.08 \pm 16.56$ & $223.75 \pm 24.19$ & $230.25 \pm 20.33$ \\
\hline
\end{tabular}

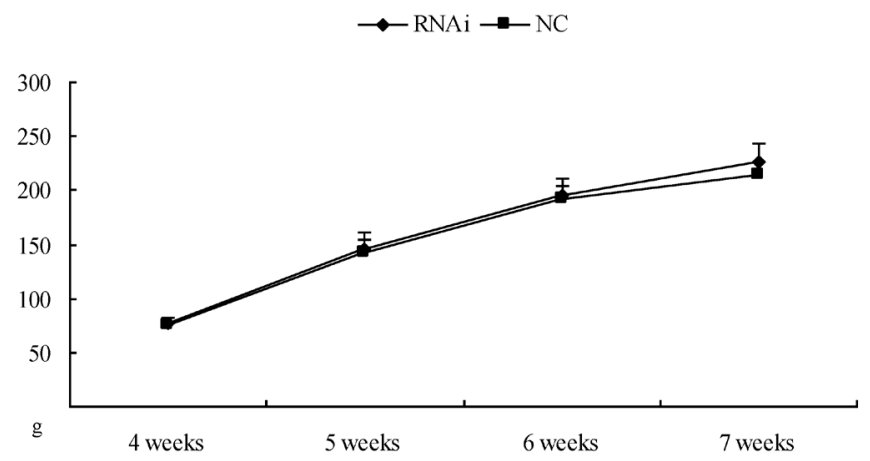

Figure 2. Body weight curve of rats after intracerebroventricular injection of Homer la-RNAi-LV (RNAi) or negative control NC-LV (NC). No difference was observed between RNAi group and $\mathrm{NC}$ group (4 weeks: $t=0.18, P>0.05 ; 5$ weeks: $t=0.62, P>0.05$; 6 weeks: $t=0.96, P>0.05 ; 7$ weeks: $t=0.57, P>0.05)$.

\section{Behavioral assessments}

A Làt maze was used before and after intracerebroventricular injection of lentivirus to evaluate the behavior changes on both the locomotor activity and non-selective attention (NSA) of rats due to down-regulation of Homer 1a expression by RNAi compared to the negative control. The Làt maze [18] consisted of a $60 \times 60 \times 40 \mathrm{~cm}$ black plastic box with a $30 \times 30 \times 40 \mathrm{~cm}$ transparent smaller box inserted in the middle. Rats were allowed to explore the resulting corridor (60 cm long, $15 \mathrm{~cm}$ wide and $40 \mathrm{~cm}$ high). The experimental box was illuminated by a white, cold 4-W lamp placed $60 \mathrm{~cm}$ above the floor in the center, providing illumination of $0.1-0.2 \mathrm{~mW} / \mathrm{cm}^{2}$. All lights except the cold lamp were turned off at the beginning of the experiment. The behavior was monitored using a CCD camera, and a set of infrared photocells was fixed surrounding the box to detect rearing. Horizontal (ambulatory) and vertical (rearing) activities were defined as the distances the rats traveled as recorded by a video tracking system and the number of times the rats stood upright on their hind limbs, respectively. The distance of individual ambulatory in the Làt maze was used to evaluate the locomotor activity levels. The frequency or duration of individual rearing episodes on the hindlimbs by rats in a spatial novelty situation has been shown to index NSA. Here we used frequency of rearing in the fixed time in the Làt maze to evaluate the NSA level [19].

A Morris water maze was used to evaluate the behavior differences on spatial learning in rats due to down-regulation of Homer 1a expression by RNAi compared to the negative control. The Morris water maze consisted of a cylindrical tub $(130 \mathrm{~cm}$ diameter, $50 \mathrm{~cm}$ depth) that was filled with water $\left(23 \pm 2^{\circ} \mathrm{C}\right)$ to $11 \mathrm{~cm}$ below the rim. The water was rendered opaque by the addition of black, non-toxic paint. The pool was divided into four quadrants of equal areas, which were arbitrarily called northeast, southeast, southwest and northwest. A circular platform $(9 \mathrm{~cm}$ diameter $)$ was submerged $1 \mathrm{~cm}$ below the water surface in the middle of one quadrant (the target quadrant) with its center located $30 \mathrm{~cm}$ from the perimeter of the maze. A closed-circuit television camera mounted onto the ceiling directly above the center of the pool was used to record swim trajectories and other parameters, which were then processed by an electronic image analyzer (HVS Image Ltd., Twickenham, Middlesex, UK). Testing was conducted in two phases: cued and acquisition. Cued trials tested the animals' ability to see and use proximal cues. Animals underwent an acquisition trial consisted of 3 trials/day for 3 con- 
secutive days as they learned to find a hidden platform that was consistently placed in one quadrant of the maze. Animals began each trial in a different cardinal position of the maze, requiring the use of constant extra maze spatial cues to find the platform. A maximum latency of $60 \mathrm{sec}$ to find the platform was allotted for each trial, and there was a 30 min rest period between trials. Rats that failed to locate the platform within the allotted time were manually guided to it and placed on the platform. All rats were allowed to remain on the platform for $30 \mathrm{sec}$ at the conclusion of each trial. A daily latency for each rat was calculated as the average of the 3 acquisition trials from 3 different directions for that day. Latency and the total swim distance were used to evaluate spatial learning ability [20].

\section{Quantitative real-time RT-PCR}

Expression level of Homer $1 a$ and Homer $1 b / c$ mRNA were detected, with actin as a normalizing control, using the following specific PCR primers designed by Primer premier 3.0 software: Homer 1a forward: 5'-TGATAGCCGGGCAAACACT-3'; Homer 1a reverse 5'-TCCTCCTGCTGATTCCTGTG-3'; Homer 1b/c forward: 5'-TTGTCTGCCTCGTTGA GTTG-3'; Homer 1b/c reverse: 5'-TAGGTTGTTCC CCCATTTTG-3'; actin forward: 5'-TCCTTCCTGGG CATGGAGT-3'; actin reverse: 5'-CAGGAGGAG CAATGATCTTGAT-3'. After completion of the real-time quantitative PCR, the amplification product was immediately conducted electrophoresis in the agarose gel. Confirm the target gene according to the position in the DNA marker. Total RNA was isolated using Trizol reagent (Invitrogen, USA), and cDNA was acquired according to the Roche procedures (Roche, Switzerland) with $2 \mu \mathrm{g}$ of total RNA. Two-step qPCR reactions were performed using the ABI 7500 Real-time PCR system (ABI, USA), and the conditions were $50^{\circ} \mathrm{C}$ for $2 \mathrm{~min}$; 40 cycles of $95^{\circ} \mathrm{C}$ for $10 \mathrm{~min}, 95^{\circ} \mathrm{C}$ for $15 \mathrm{~s}$; and a final hold at $60^{\circ} \mathrm{C}$ for 1 min. Absorbance data were collected at the end of every extension, and cycle threshold $(\mathrm{Ct})$ values were used to analyze expression data by the $2^{-\Delta \Delta \mathrm{Ct}}$ method.

\section{Western blot}

Cytoplasmic proteins were extracted from cells or tissues by using the NuclearCytosol Extraction Kit KGP150 (KeyGEN, CA). 1ml tissue was homogenized in ice-cold PBS and centrifuged at $500 \mathrm{~g}$ for $3 \mathrm{~min} .20$ $\mathrm{uL}$ tissue was resuspended in $200 \mathrm{uL}$ ice-cold lysis Buffer A (10mM Hepes, pH7.9, 500mM NaCl, 0.1mM EDTA, 0.1mM EGTA), per ml Buffer A added 1uL dithiothreitol (DTT), $0.5 \mathrm{uL}$ phenylmethylsulfonyl fluoride (PMSF), 1 uL protease inhibitors), surged 15sec and incubated for 10-15 min., then added 11 uLBufferB(10\% NP-40), surged 5sec and incubated for 10-15 min. The lysate was centrifuged at $16,000 \mathrm{~g}$ for5 $\mathrm{min}$. The supernatant was transferred into ice-cold sample tubes and stored at $-80{ }^{\circ} \mathrm{C}$ until use. Protein concentrations were determined using the BCA assay. Equal amounts of protein $(100 \mu \mathrm{g}$ in $20 \mu \mathrm{l}$ per lane) were then separated by sodium dodecyl sulfate polyacrylamide gel electrophoresis (SDS/PAGE) (Invitrogen, USA). Proteins were subsequently transferred to a polyvinylidene fluoride (PVDF) membrane (Bio-Rad, Hercules, CA) in standard transfer buffer (25 mM Tris, $192 \mathrm{mM}$ glycine, and $20 \%$ methanol).and exposed to blocking buffer (5\% nonfat dry milk in PBS with $0.1 \%$ Tween-20) at room temperature for $1 \mathrm{~h}$. The blots were incubated overnight at $4^{\circ} \mathrm{C}$ with the following primary antibodies in buffer containing bovine serum albumin: goat anti-rat Homer 1a (1:100 dilution; catalog number sc-8922, Santa Cruz Biotechnology, Santa Cruz, CA, USA) or Homer 1b/c (1:100 dilution; catalog number sc-8923, Santa Cruz)[21,22] or rabbit anti-rat $\beta$-actin $(1: 2,000$ dilution; Beijing Biosynthesis Biotechnology, Beijing, China). The membrane was then washed with TBST for $5 \mathrm{~min}$. This step was repeated 3 times. Subsequently, the blots were incubated for $1 \mathrm{~h}$ at room temperature with the following secondary antibodies: donkey horseradish peroxidase-conjugated anti-goat antibody (1:2,000 dilution; Santa Cruz Biotechnology) for Homer 1a and Homer 1b/c or goat anti-rabbit antibody (1:2,000 dilution; Santa Cruz) for $\beta$-actin. The membrane was then washed with TBST for $5 \mathrm{~min}$. This step was repeated 3 times. Bands were visualized with Western Lightning ECL (Perkin-Elmer Life Sciences, Inc., Waltham, Massachusetts, USA) and analyzed using Image Lab version 2.0.1 (Bio-Rad) and quantified by densitometry or by quantification on a Kodak Image Station 2000R (Eastman Kodak/SIS). Density values of the Homer 1a or Homer 1b/c protein bands were each expressed as a percentage of the density of the $\beta$-actin internal standard.

\section{Statistical analyses}

Statistical analysis of the data involving three groups for in vitro validation was performed using one way ANOVA, and post hoc tests (LSD or SNK) were used following the one way ANOVA. While the data involving two groups for in vivo validation was analyzed using an independent samples t-test. For all analyses, significance was assigned at the $P \leq 0.05$ level. All data are presented as the means \pm standard deviation (S.D.) 


\section{Results}

\section{Homer I a-RNAi-LV down-regulates expres- sion of Homer I a mRNA and protein in the primary neural cells}

The primary neural cells obtained from the rat's cortices was cultured for two days and then transduced with the lentiviral vector containing Homer 1a-RNAi-LV or NC-LV at the appropriate MOI determined pre-experimentally. The expression levels of Homer 1a mRNA and protein were then evaluated using qPCR and Western blot. After 72 hours transduction with Homer 1a-RNAi-LV, the primary neural cells showed a significant reduction in the level of Homer 1a mRNA expression (vs. control groups, $\mathrm{F}=5.93, P<0.05$ ), as well as protein expression (vs. control groups, $\mathrm{F}=6.40, P<0.05$ ) (see Table 3 , Figure 3 ). Therefore, Homer 1a was successfully down-regulated in cultured neural cells after RNAi using a lentiviral vector containing an artifical Homer 1a-specific miRNA.

\section{Effects of Homer la-RNAi-LV on behaviors of SD rats}

24 SD rats were divided into two groups of 12 in each and given Homer 1a-RNAi-LV (RNAi group) or NC-LV (NC group) by intracerebroventricular injection to examine the effects of Homer 1a RNAi on behaviors in the Làt maze and the Morris water maze tests. Prior to intracerebroventricular injection of the lentivirus vectors, no significant difference was found in the horizontal activities and frequency of rearings of the two groups in the Làt maze (horizontal activities: $t=0.01, P>0.05$; frequence of rearings: $t=0.16$, $P>0.05)$. Seven days after intracerebroventricular injection of lentiviruses, rats given Homer 1a-RNAi-LV (RNAi rats) exhibited significantly higher levels of both horizontal activities and frequency of rearings in the Làt maze than the control rats given NC-LV (NC rats) (horizontal activities: $\mathrm{t}=2.41, P<0.05$; frequence of rearings: $t=2.37, P<0.05$ ) (see Table 4 , Figure 4 ). After three days of training in the Morris water maze, RNAi group rats performed significantly worse in the Morris water maze than the NC group rats, as displayed by longer latency times before locating the platform and longer total swim distances from the first day to the third day, but only on the third day the results were statistically significant (D1, latency: $t=1.46$, $P=0.16$, distance: $\mathrm{t}=0.82, P=0.42 ; \mathrm{D} 2$, latency: $\mathrm{t}=2.00$, $P=0.06$, distance: $\mathbf{t}=2.05, P=0.05$; $\mathrm{D}$, latency: $\mathbf{t}=2.95$, $P=0.01$, distance: $\mathbf{t}=3.75, P=0.01$ ) (see Table 5, Figure $5)$. Therefore, in this study the RNAi group displayed a range of abnormal behaviors. First, significantly higher levels of both horizontal activities and frequency of rearings in the Làt maze were observed, which meant the locomotor hyperactivity and NSA of SD rats were increased after Homer 1a RNAi. Second, these rats showed longer latencies before locating the platform and longer total swim distances, which indicated that the learning ability of rats was damaged after Homer 1a down-regulation.

Table 3 Reduction of Homer la mRNA and protein expression by Homer la-RNAi-LV transduction in the primary cultured neural cells.

\begin{tabular}{llll}
\hline & $\mathrm{Ctr}$ & $\mathrm{NC}$ & $\mathrm{RNAi}$ \\
\hline mRNA & $1.00 \pm 0.43$ & $1.00 \pm 0.04$ & $0.35 \pm 0.16^{*}$ \\
protein & $1.00 \pm 0.25$ & $0.92 \pm 0.19$ & $0.49 \pm 0.10^{*}$ \\
\hline
\end{tabular}

* $P<0.05$, Ctr, non-transduced cells; NC, NC-GFP-LV group; RNAi, Homer 1a-RNAi-LV transduced group.

A

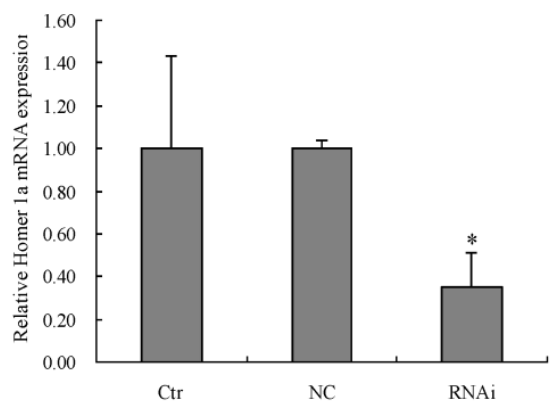

$\mathrm{B}$

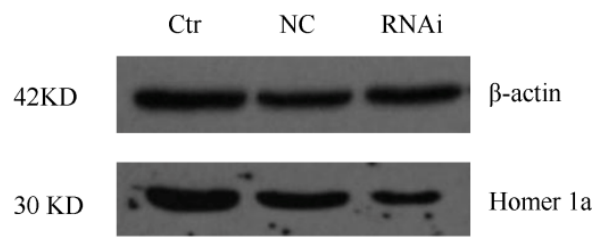

Figure 3. Reduction of Homer Ia mRNA and protein expression by Homer la-RNAi-LV transduction in the primary cultured neural cells. Ctr, non-transduced cells; NC, NC-GFP-LV group; RNAi, Homer Ia-RNAi-LV transduced group (A) Homer I a mRNA expression decreased significantly in cells transduced with Homer la-RNAi-LV (vs. control groups, $F=5.93, P<0.05$ ). (B) By Western blot, Homer la protein expression decreased significantly in cells transduced with Homer la-RNAi-LV (vs. control groups, $F=6.40, P<0.05$ ). Data are expressed as means \pm SD. $n=3$. $* P<0.05$. 
Table 4. Locomotor activity levels and non-selective attention in Làt maze test of rats before and after intracerebroventricular injection of Homer la-RNAi-LV (RNAi) or negative control NC-LV (NC)

\begin{tabular}{llll}
\hline Group & Before RNAi & \multicolumn{2}{l}{ After RNAi } \\
\cline { 2 - 4 } & horizontal activities $(\mathrm{mm})$ & rearing frequency & horizontal activities $(\mathrm{mm})$ \\
\hline NC $(\mathrm{n}=12)$ & $63456.62 \pm 9225.86$ & $61.08 \pm 21.52$ & $53700.48 \pm 8010.11$ \\
RNAi $(\mathrm{n}=12)$ & $63473.94 \pm 7466.82$ & $62.42 \pm 18.98$ & $61865.2 \pm 8583.27^{*}$ \\
\hline
\end{tabular}

${ }^{*} P<0.05$.
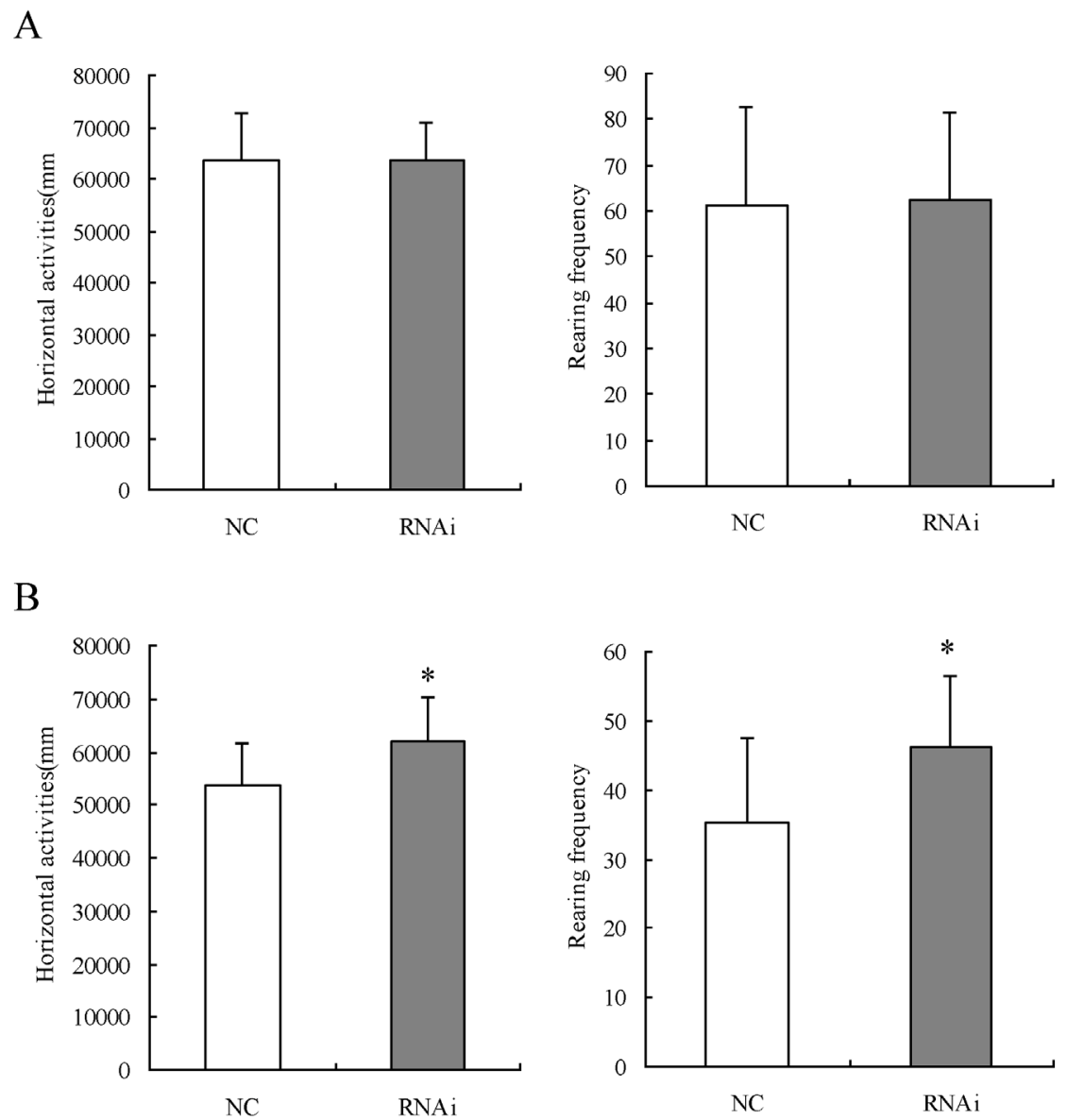

Figure 4. Locomotor activity levels and non-selective attention in Làt maze test of rats before and after intracerebroventricular injection of Homer Ia-RNAi-LV (RNAi) or negative control NC-LV (NC). (A) No significant difference was found in the horizontal activities and frequency of rearings of the two groups in the Làt maze (horizontal activities: $t=0.0 I, P>0.05$; frequence of rearings: $t=0.16, P>0.05$ ). $(B$ ) Seven days after intracerebroventricular injection of lentiviral vectors, RNAi rats exhibited significantly higher levels of both horizontal activities and frequency of rearings in the Làt maze than NC rats (horizontal activities: $t=2.4 I, P<0.05$; frequence of rearings: $t=2.37$, $P<0.05)$. Data are expressed as means \pm SD. $n=12 . * P<0.05$.

Table 5. Spatial learning performance in Morris water maze of rats after intracerebroventricular injection of Homer la-RNAi-LV (RNAi) or negative control NC-LV (NC).

\begin{tabular}{lllllll}
\hline Group & \multicolumn{5}{l}{ total swim distances $(\mathrm{mm})$} & \multicolumn{3}{l}{ Latency $(\mathrm{s})$} \\
\cline { 2 - 6 } & $\mathrm{D} 1$ & $\mathrm{D} 2$ & $\mathrm{D} 3$ & $\mathrm{D} 1$ & $\mathrm{D} 2$ & D3 \\
\hline Nc $(\mathrm{n}=12)$ & $17159.04 \pm 5749.81$ & $9133.38 \pm 4192.88$ & $6252.57 \pm 2200.11$ & $138.25 \pm 47.59$ & $82.42 \pm 33.20$ & $57.75 \pm 25.40$ \\
RNAi $(\mathrm{n}=12)$ & $15267.06 \pm 5506.77$ & $12560.77 \pm 3985.62$ & $11237.97 \pm 4050.40$ & $167.17 \pm 49.16$ & $110.35 \pm 35.90$ & $91.58 \pm 30.51$ \\
\hline
\end{tabular}

${ }^{*} \mathrm{P}<0.05$ 
A

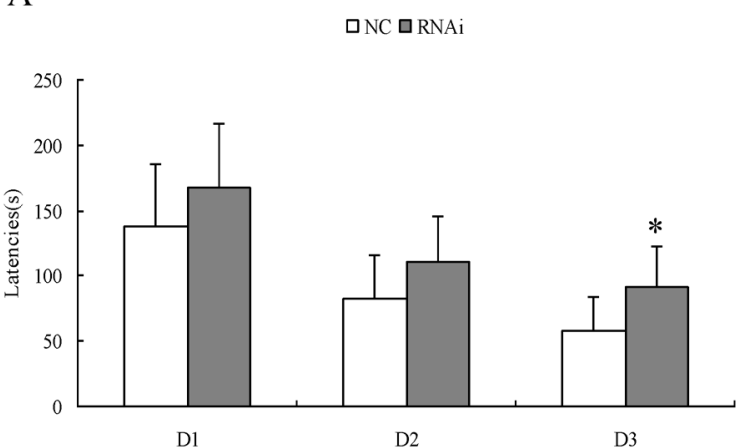

B

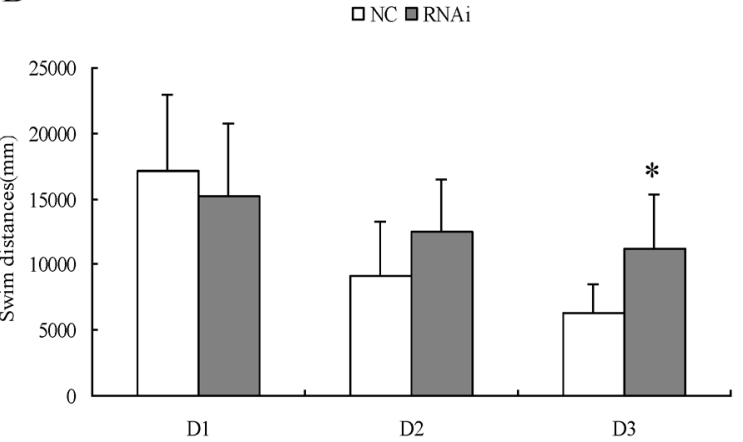

Figure 5. Spatial learning performance in Morris water maze of rats after intracerebroventricular injection of Homer la-RNAi-LV (RNAi) or negative control NC-LV (NC). D I-3, days I-3 of training. (A) RNAi rats showed significantly longer latencies before locating the platform on the third day (DI, $\mathrm{t}=1.46, P=0.16$; 2 2, $\mathrm{t}=2.00, P=0.06 ; \mathrm{D} 3, \mathrm{t}=2.95, P=0.0 \mathrm{I}$ ). (B) RNAi rats had significantly longer total swim distances on the third day (DI, $\mathrm{t}=0.82, P=0.42 ; \mathrm{D} 2, \mathrm{t}=2.05, P=0.05 ; \mathrm{D} 3, \mathrm{t}=3.75, P=0.0 \mathrm{I})$. Data are expressed as means $\pm \mathrm{SD}$. $\mathrm{n}=12$. $* P<0.05$.

\section{Effects of Homer Ia-RNAi-LV on the mRNA and protein expression of Homer I a and Homer Ib/c}

After completing the behavioral assessments, we randomly selected four rats from each of the two groups. The mRNA and protein expression level of Homer 1a and Homer 1b/c were detected by qPCR and Western blot, respectively. As shown in Table 6/Table 7 and Figure 6, significantly decreased Homer $1 a$ mRNA expression level in the striatum and was observed in the hippocampus of RNAi group rats compared with those of the NC group (striatum: $t$ $5.25, P<0.01$; hippocampus: $t=5.80, P<0.01)$. However, we found no significant difference between the groups with respect to Homer $1 a$ mRNA expression in the prefrontal cortex $(\mathrm{PFC})(\mathrm{t}=1.82, P>0.05)$. Western blotting further revealed the significant effect of Homer 1a-RNAi-LV on Homer 1a protein expression in the striatum and hippocampus (striatum: $\mathrm{t}=3.95$, $P<0.05$; hippocampus: $t=3.48, P<0.05)$, while there was no effect in the PFC ( $t=1.03, P>0.05)$. Meanwhile, no significant difference between the groups with respect to Homer $1 b / c$ mRNA or protein expression was found in the striatum, hippocampus or PFC (mRNA: striatum: $\mathrm{t}=1.02, P>0.05$; hippocampus: $\mathrm{t}=0.45, \quad P>0.05$ : PFC: $\mathrm{t}=1.02, P>0.05$; protein: striatum: $\mathrm{t}=0.87, P>0.05$; hippocampus: $\mathfrak{t}=0.18, P>0.05$ : PFC: $\mathfrak{t}=0.62, P>0.05$ ). Therefore, the RNAi group, which displayed a range of abnormal behaviors, exhibited a significant decrease in Homer 1a expression in the striatum and hippocampus compared to the NC group.

Table 6. mRNA and protein expression of Homer la in rats after intracerebroventricular injection of Homer la-RNAi-LV (RNAi) or negative control NC-LV (NC).

\begin{tabular}{lllllll}
\hline & mRNA & \multicolumn{5}{l}{ protein } \\
\cline { 2 - 6 } & Str & Hip & PFC & Str & Hip & PFC \\
\hline NC & $1.00 \pm 0.10$ & $1.00 \pm 0.07$ & $1.00 \pm 0.23$ & $1.00 \pm 0.17$ & $1.00 \pm 0.27$ & $1.00 \pm 0.21$ \\
RNAi & $0.45 \pm 0.19^{* *}$ & $0.59 \pm 0.12^{* *}$ & $0.75 \pm 0.16$ & $0.52 \pm 0.18^{* *}$ & $0.51 \pm 0.07^{* *}$ & $0.84 \pm 0.22$ \\
\hline
\end{tabular}

$* * P<0.01$

Table 7. mRNA and protein expression of Homer $\mathrm{Ib} / \mathrm{c}$ in rats after intracerebroventricular injection of Homer Ia-RNAi-LV (RNAi) or negative control NC-LV (NC).

\begin{tabular}{lllllll}
\hline & \multicolumn{5}{c}{ protein } \\
\cline { 2 - 7 } & Str & Hip & PFC & Str & Hip & PFC \\
\hline NC & $1.00 \pm 0.13$ & $1.00 \pm 0.35$ & $1.00 \pm 0.11$ & $1.00 \pm 0.07$ & $1.00 \pm 0.13$ & $1.00 \pm 0.20$ \\
RNAi & $0.89 \pm 0.19$ & $0.91 \pm 0.27$ & $1.14 \pm 0.24$ & $1.04 \pm 0.06$ & $1.01 \pm 0.07$ & $0.94 \pm 0.09$ \\
\hline
\end{tabular}


A

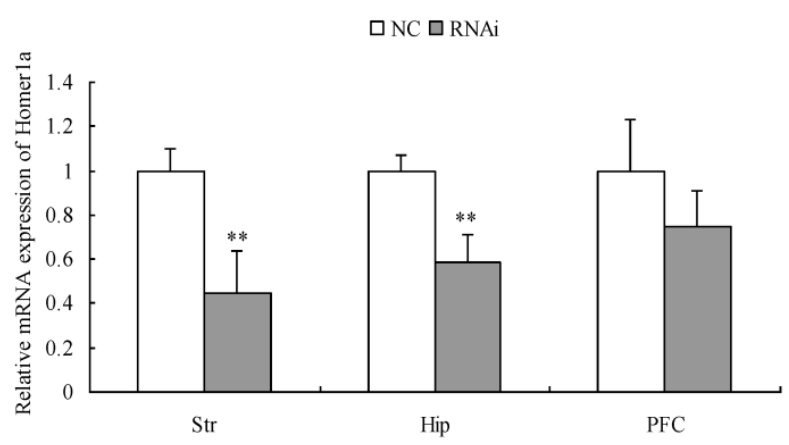

C

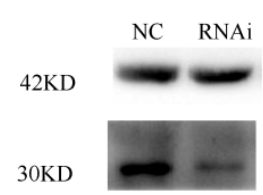

Str

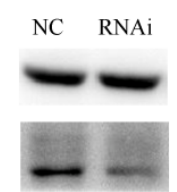

Hip

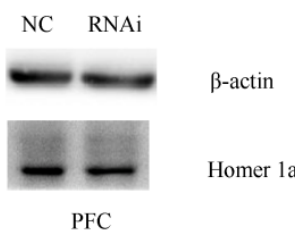

B

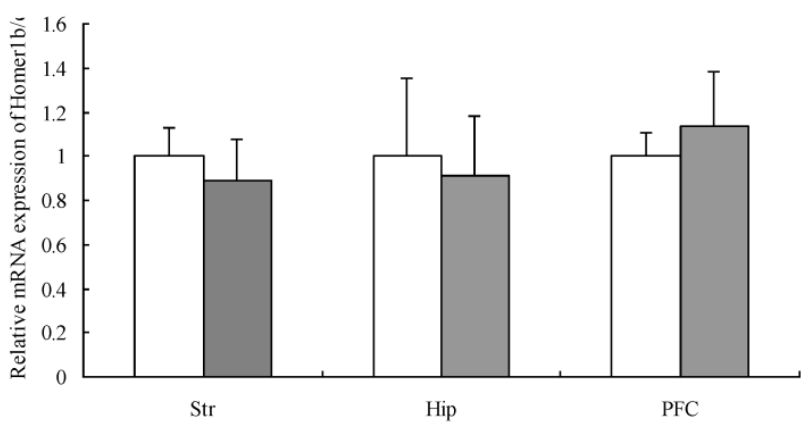

D

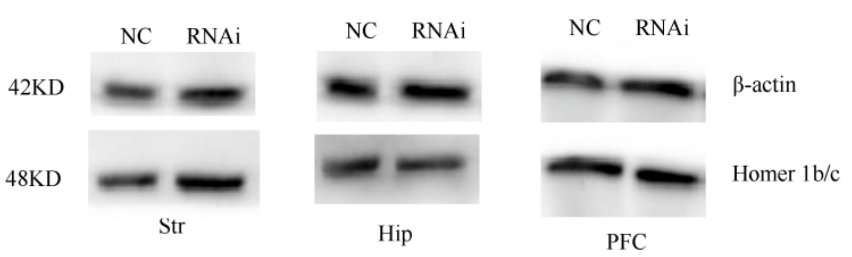

口NC QRNAi

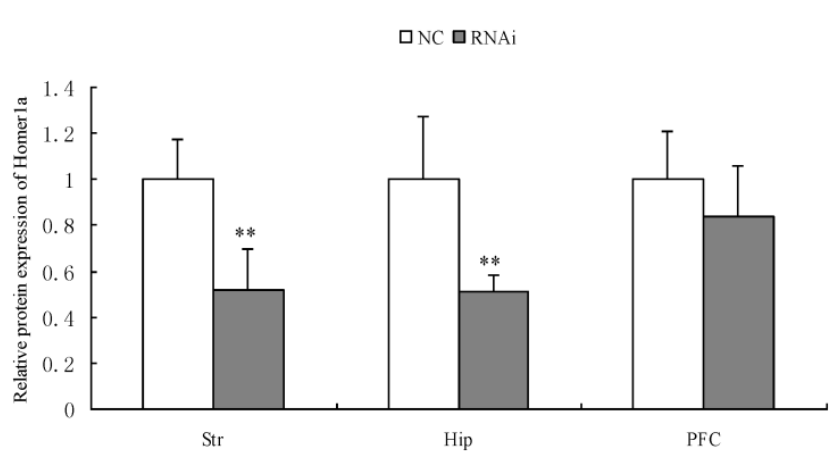

口NC Q RNAi

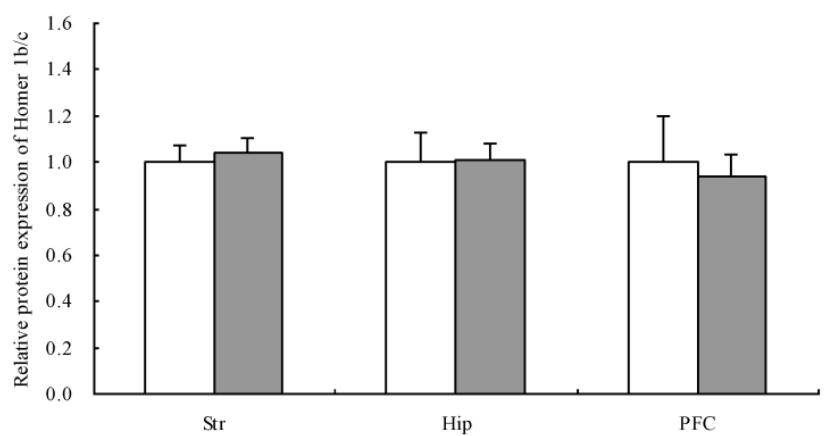

Figure 6. $m R N A$ and protein expression of Homer la in rats after intracerebroventricular injection of Homer la-RNAi-LV (RNAi) or negative control NC-LV (NC). (A) RNAi rats showed significantly decreased Homer la mRNA level in the striatum $(\mathrm{Str})(\mathrm{t}=5.25, \mathrm{P}<0.05)$ and hippocampus (Hip) $(t=5.80, P<0.05)$ vs. NC group, but no difference in the prefrontal cortex (PFC) $(t=1.82, P>0.05)$ vs. NC group. (B) No difference of Homer Ib/c mRNA in the striatum (Str) $(t=I .02, P>0.05)$, the hippocampus (Hip) $(t=0.45, P>0.05)$ and the prefrontal cortex (PFC) $(t=1.02, P>0.05)$ was observed between the RNAi group and the NC group. (C) RNAi rats showed significantly decreased Homer I a protein level in the striatum $(\mathrm{Str})(\mathrm{t}=3.95, \mathrm{P}<0.05)$ and hippocampus $(\mathrm{Hip})(\mathrm{t}=3.48, \mathrm{P}<0.05)$ vs. NC group, but no difference in the prefrontal cortex (PFC) $(t=1.03, P>0.05)$ vs. NC group. (D) No difference of Homer Ib/c protein in the striatum (Str) ( $t=0.87$, $P>0.05)$, the hippocampus (Hip) $(t=0.18, P>0.05)$ and the prefrontal cortex (PFC) $(t=0.62, P>0.05)$ was observed between the RNAi group and the NC group. Data are expressed as means $\pm S D . n=4$. $* * P<0.01$.

\section{Discussions}

\section{In vitro and in vivo validation of the lentiviral vector containing an artificial Homer la spe- cific miRNA as an RNAi tool}

Homer $1 a$ was a down-regulated target gene screened out by gene expression profiling in the
ADHD animal model and verified by RT-PCR and Western blot in our previous studies [2-4]. In this study we investigated the functional relationship between Homer 1a and ADHD using RNAi. Homer 1a was successfully down-regulated not only in cultured neural cells but also in the brain of SD rats after RNAi using a lentiviral vector containing an artifical Homer 1a-specific miRNA. 
RNAi has recently emerged as a useful genetic method for silencing gene expression by triggering post-transcriptional degradation of homologous transcripts and has become an invaluable tool for understanding diverse biological processes, both normal and pathological. We first screened for the most efficient RNAi target sequence among the four candidate miRNAs using $\mathrm{H} 9 \mathrm{c} 2$ cells. A Gateway-adapted expression vector that enables the expression of engineered miRNA sequences from Pol II promoters was chosen in this study instead of siRNA or shRNA [23-25]. Our results showed that the engineered miRNAs fully complemented their target site and cleaved the target mRNA. As our target gene was in the brain of rats, we needed a vector that could introduce the miRNA sequence into nerve cells and sustain the knockdown effect for a sufficient amount of time for molecular and behavior assessments. Lentvirus vector-mediated expression of RNAi can achieve effective, stable gene silencing in biological systems, with the reason of that the objective gene is integrated into the host cell genome, and is divided with the splitting of the cell's genome. In addition, lentiviral vector can efficiently infect and integrate into non-dividing cells [26,27]. Therefore, we chose such a system to package the above target vector for transfer into primary cultured neural cells, to further verify the interference efficiency in vitro. The results showed that lentivirus mediated RNAi could effectively transduce primary cultured neural cells. Furthermore, this lentiviral vector was further transferred to the brain tissue of rats by intracerebroventricular injection to verify the interference efficiency in vivo. The results indicated that was Homer 1 a expressed at a lower level in the striatum and hippocampus of RNAi rats. All these results indicated that lentivirus-mediated miRNA was an effective RNAi way of modulating Homer 1a expression not only in vitro but also in vivo, which was important to validate before subsequent experiments. However, no change in expression of Homer 1a was found in the PFC. We measured the range of the viral infection using immunofluorescence 7 days after the injection. The florescence of striatum and hippocampus were strong and full vision distribution, while it was scattered in prefrontal cortex. We speculate that the distance between the structures and the lateral ventricle affected the viral tranduction efficiency. The striatum and hippocampus are anatomically located more closely to the lateral ventricle than the PFC. Although it is possible that the PFC could have been infected by the injected lentiviral vector via circulating cerebrospinal fluid, any effect would have been weakened. Maybe infusing virus directly into the PFC will be helpful for us to research the behaviors of the rats with the detection of Homer 1a in this region in the future.

\section{Effect of down-regulation of Homer I a ex- pression by RNAi on behaviors of SD rats}

In this study we found that the RNAi group, which exhibited a significant decrease in Homer 1a expression in the striatum and hippocampus compared to the NC group, displayed a range of abnormal behaviors. First, significantly higher levels of both horizontal activities and frequency of rearings in the Làt maze were observed, which meant the locomotor hyperactivity and NSA of SD rats were increased after Homer 1a RNAi. Attentive processes can be of the selective (SA) or non-selective (NSA) type. The major pathology of attentive processes is represented by ADHD that is characterised by attentional problems accompanied or not by hyperkinesis, compulsiveness, restlessness, and disturbances in timing (DSM-IV [28]. The frequency and duration of individual rearing episodes on the hindlimbs by rats in a spatial novelty situation has been shown to index NSA $[18,19]$. In fact, the animal model of ADHD, such as SHR and NHE display high frequency of rearings which is interpreted to reflect defective NSA, since it leads eventually to impaired long term behavioral habituation to novelty (cognitive defect)[18,19]. Therefore, the behaviors of the rats after Homer 1a RNAi was similar to that of the animal model of ADHD in Làt maze. Second, these rats showed longer latencies before locating the platform and longer total swim distances in the acquisition trials, which indicated that the learning ability of rats were damaged after Homer 1a down-regulation. When placed in the maze the animal's task is to find the hidden platform, the experimenter can chart the "learning" of the animal by the time it takes to find the platform over a number of trials. However, there are many alternative explanations for this hypothetical data set, such as decreased vision or anxiety in the water, which could impair the animals' ability to learn the task or decreased the desire to escape from the water, respectively. Therefore, a cued trial was performed between the RNAi and NC groups before acquisition trial. No difference was found in latencies between the two groups, which mean the animals' vision and the motivation to escape the water were not impaired in this study [29]. Neither a probe trial nor a reversal of platform location was conducted in the water maze trials of the current study. Such information may have been helpful in further interpretations of the difference between the RNAi and NC rats [30]. All these abnormal behaviors (increased locomotor and NSA, impaired learning) are reminiscent of ADHD [28]. 
Fronto-striatal, fronto-cerebellar and fronto-parietal neural networks disorders have been reported in ADHD. The striatum is best known for its role in the planning and modulation of movement pathways, but it is also involved in a variety of other cognitive processes involving executive function. Meanwhile, it is activated by stimuli associated with reward. The important role of the striatum in ADHD has been illustrated by neuroimaging studies showing decreases in blood flow in regions of the striatum [31], and changes in dopamine transporter binding [32] have been described in the human striatum in ADHD. In a number of paradigms, functional magnetic resonance imaging (fMRI) techniques have shown reduced activation in prefrontal and striatal regions in ADHD during specific cognitive and behavioral tasks as well as reduced activation of the ventral striatum in reward anticipation relative to controls [33]. Neuropsychological research also has revealed prefrontal-striatum defects in ADHD, including executive functioning defects mediated by fronto-striatal, fronto-cerebellar and fronto-parietal neural networks [34]. In addition, the role of the hippocampus in ADHD is gaining increased attention. A widely accepted hypothesis for ADHD involves dysfunction of the PFC [35-37], which is connected directly to the ventral hippocampus and indirectly to the dorsal hippocampus via the thalamus. The PFC-hippocampal circuit regulates a variety of processes that have been implicated in the pathophysiology of ADHD, including attention, memory and emotion [38-40]. For instance, Plessen et al. found that children and adolescents with ADHD have larger hippocampal volumes than healthy controls, primarily due to increases in the anterior portions of the hippocampus. As larger hippocampal volumes tend to be associated with less severe ADHD symptoms [41], the enlarged anterior portions of the hippocampus may represent neural compensation for dysfunctional PFC activity. In addition, rats with X-ray-induced hippocampal damage show cognitive improvement in response to amphetamine, a stimulant used to treat ADHD [42]. As Homer $1 a$ was a down-regulated target gene screened out by gene expression profiling in the ADHD animal model and verified by RT-PCR and Western blot in our previous studies [2-4] and the rats become hyperactivity, attention deficit and learning impaired after Homer 1a RNAi. Therefore, we speculated that the effects of Homer 1a down-regulation in these brain structures are related to movement, executive function and learning ability, thereby causing certain behavioral changes similar to ADHD. However, we believe that there are still something more need to do to find the positive correlation between the levels of
Homer-1a silencing and the behavioral performance of animals.

\section{Cellular mechanisms involved in the behavior changes of SD rats induced by Homer Ia RNAi}

As a short isoform, Homer 1a is functionally different from long-form Homer proteins, because it lacks the C-terminal coiled-coil structure and leucine zipper motifs. In basal conditions, long-form Homer proteins homomultimerize with these structures and bind the C-terminus of mGluR I (mGluR1, mGluR5) via the N-terminal EVH1 (Enabled/VASP homology 1) domain. In detail, long-form Homer proteins bridge mGluRs I with intracellular Ca2+ storage pools by interacting with IP3Rs and possibly with ryanodine receptors (RyRs) [43]. Thus, as a prominent scaffolding molecule concentrated in the PSD of excitatory synapses, Homer crosslinks group I mGluRs to other targets in a specific subcellular microdomain to regulate a specific signaling activity. Moreover, long-form Homers form a quaternary complex, Homer-Shank-GKAP-PSD95, that crosslinks mGluRs I with NMDA-Rs, and the interaction of Homer with Shank is involved in cytoskeleton rearrangement within the PSD [44,45]. Although Homer 1a lacks the structure for self-assembly, it can compete with long-form Homers to bind ligands, thus behaving as a dominant negative. Homer 1a disrupts the preformed bridge connecting mGluRs I with IP3Rs, and the quaternary complex cross-linking mGluRs I with NMDA-Rs [45]. Moreover, Homer 1a, as an immediate early gene, is constitutively expressed at a low level, but is highly inducible in response to stimulation $[46,47]$. These actions cause rapid changes in intracellular $\mathrm{Ca} 2+$ and may modulate PSD remodeling. While the PSD is a specialized ultrastructure of the glutamatergic excitatory synapses, at the cytosolic surface of the postsynaptic membrane of dendritic spines is a highly organized transductional machinery that tunes the excitatory signaling from presynaptic terminals [48]. Therefore, Homer 1a plays a critical role in modulating excitatory post-synaptic signal transduction. In addition, due to the involvement of mGluRs I in multiple transduction pathways through activation of a broad range of intracellular effectors, such as adenylate cyclase, tyrosine-kinase or mitogen-activated protein kinase (MAPK), mGluRs I have been linked to synaptic plasticity, neuronal development [49-51] and pathological conditions such as epilepsy or Parkinson's disease [52-54]. The down-regulation of Homer 1a expression by RNAi may reduce functional targeting of mGluRs to the synaptic membrane, decreasing the fidelity of synaptic transmission through affecting the clustering, tar- 
geting and functional activity of neurotransmitter receptors by synaptic density proteins. Therefore, in future work, we will further explore the expressions of the long-term Homer and some related genes like mGluRs and Shank to investigate if the significantly lowered expression of Homer 1a may affect modulation of excitatory post-synaptic signal transduction or the plastic dendritic remodeling of the striatum and hippocampus, thereby producing a series of ADHD-like behavior changes.

In conclusion, down-regulation of Homer 1a resulted in some behaviors similar to ADHD in rats. All of these will help us gain a better understanding of the role of these molecules in neurological changes in ADHD.

\section{Acknowledgments}

This study was supported by grants from the National Natural Science Foundation of China (No. 30801255, 81250008), the Medical Priority Talents of Jiangsu Province, China (No. RC2011022), and Nanjing Medical Science and Technology Development Project, Jiangsu Province, China (No. 201108007).

\section{Competing Interests}

The authors have declared that no competing interest exists.

\section{References}

1. Katragadda S, Schubiner H. ADHD in children, adolescents, and adults. Prim Care. 2007;34:317-341.

2. Qiu J, Hong Q, Chen RH, et al. Gene expression profiles in the prefrontal cortex of SHR rats by cDNA microarrays. Mol Biol Rep. 2010;37:1733-1740.

3. Hong Q, Wang $\mathrm{YP}$, Zhang $\mathrm{M}$, et al. Homer expression in the hippocampus of an animal model of attention-deficit/hyperactivity disorder. Mol Med Report. 2011;4:705-712.

4. Hong Q, Zhang M, Pan XQ, et al. Prefrontal cortex Homer expression in an animal model of attention-deficit/hyperactivity disorder. J Neurol Sci. 2009;287:205-211.

5. de Bartolomeis A, Iasevoli F. The Homer family and the signal transduction system at glutamatergic postsynaptic density: potential role in behavior and pharmacotherapy. Psychopharmacol Bull. 2003;37:51-83.

6. Tekin S, Cummings JL. Frontal-subcortical neuronal circuits and clinical neuropsychiatry: an update. J Psychosom Res. 2002;53:647-654.

7. Jaubert PJ, Golub MS, Lo YY, et al. Complex, multimodal behavioral profile of the Homer1 knockout mouse. Genes Brain Behav. 2007;6:141-154.

8. Szumlinski KK, Lominac KD, Kleschen MJ, et al. Behavioral and neurochemical phenotyping of Homer1 mutant mice: possible relevance to schizophrenia. Genes Brain Behav. 2005;4:273-288.

9. Lominac $\mathrm{KD}$, Oleson $\mathrm{EB}$, Pava $\mathrm{M}$, et al. Distinct roles for different Homer1 isoforms in behaviors and associated prefrontal cortex function. J Neurosci. 2005;25:11586-11594.

10. Koh S, Chung H, Xia H, Mahadevia A, Song Y. Environmental enrichment reverses the impaired exploratory behavior and altered gene expression induced by early-life seizures. J Child Neurol. 2005;20:796-802.

11. Inoue $Y$, Udo H, Inokuchi K, Sugiyama H. Homer1a regulates the activity-induced remodeling of synaptic structures in cultured hippocampal neurons. Neuroscience. 2007;150:841-852

12. Sierant M, Kubiak K, Kazmierczak-Baranska J, et al. Evaluation of BACE1 Silencing in Cellular Models. Int J Alzheimers Dis. 2009;

13. Kim VN, Han J, Siomi MC. Biogenesis of small RNAs in animals. Nat Rev Mol Cell Biol. 2009;10:126-139.
14. Daude N, Marella M, Chabry J. Specific inhibition of pathological prion protein accumulation by small interfering RNAs. J Cell Sci. 2003;116:2775-2779.

15. Kang SG, Roh YM, Lau A, et al. Establishment and characterization of Prnp knockdown neuroblastoma cells using dual microRNA-mediated RNA interference. Prion. 2011;5:93-102.

16. Amendola M, Passerini L, Pucci F, et al. Regulated and multiple miRNA and siRNA delivery into primary cells by a lentiviral platform. Mol Ther. 2009;17:1039-1052.

17. George Paxinos CW. The Rat Brain in Stereotaxic Coordinates. Third ed, New York: Academic Press; 1997.

18. Aspide R, Fresiello A, de Filippis G, Gironi CU, Sadile AG. Non-selective attention in a rat model of hyperactivity and attention deficit: subchronic methylphenydate and nitric oxide synthesis inhibitor treatment. Neurosci Biobehav Rev. 2000;24:59-71.

19. Aspide R, Gironi CU, Sergeant JA, Sadile AG. Non-selective attention and nitric oxide in putative animal models of Attention-Deficit Hyperactivity Disorder. Behav Brain Res. 1998;95:123-133.

20. Ruiz-Medina J, Morgado-Bernal I, Redolar-Ripoll D, Aldavert-Vera L, Segura-Torres P. Intracranial self-stimulation facilitates a spatial learning and memory task in the Morris water maze. Neuroscience. 2008;154:424-430.

21. Van Keuren-Jensen K, Cline HT. Visual experience regulates metabotropic glutamate receptor-mediated plasticity of AMPA receptor synaptic transmission by homer1a induction. J Neurosci. 2006;26:7575-7580.

22. Guo WG, Su FF, Yuan LJ, et al. Simvastatin inhibits angiotensin II-induced cardiac cell hypertrophy: role of Homer 1a. Clin Exp Pharmacol Physiol. 2010;37:40-45.

23. Elbashir SM, Harborth J, Lendeckel W, et al. Duplexes of 21-nucleotide RNAs mediate RNA interference in cultured mammalian cells. Nature. 2001:411:494-498.

24. Brummelkamp TR, Bernards R, Agami R. A system for stable expression of short interfering RNAs in mammalian cells. Science. 2002;296:550-553

25. Paddison PJ, Caudy AA, Bernstein E, Hannon GJ, Conklin DS. Short hairpin RNAs (shRNAs) induce sequence-specific silencing in mammalian cells. Genes Dev. 2002;16:948-958.

26. Naldini L, Blomer U, Gallay $P$, et al. In vivo gene delivery and stable transduction of nondividing cells by a lentiviral vector. Science. 1996;272:263-267.

27. Follenzi A, Ailles LE, Bakovic S, Geuna M, Naldini L. Gene transfer by lentiviral vectors is limited by nuclear translocation and rescued by HIV-1 pol sequences. Nat Genet. 2000;25:217-222.

28. American Psychiatric Association. Diagnostic and Statistical Manual of Mental Health; 4th ed. Washington, DC: American Psychiatric Association. 1994.

29. Amos-Kroohs RM, Williams MT, Vorhees CV. Neonatal methylphenidate does not impair adult spatial learning in the Morris water maze in rats. Neurosci Lett. 2011;502:152-156.

30. Ferguson SA, Cada AM. Spatial learning/memory and social and nonsocial behaviors in the spontaneously hypertensive, Wistar-Kyoto and Sprague-Dawley rat strains. Pharmacol Biochem Behav. 2004;77:583-594.

31. Lou HC, Henriksen L, Bruhn P, Borner H, Nielsen JB. Striatal dysfunction in attention deficit and hyperkinetic disorder. Arch Neurol. 1989:46:48-52.

32. Dougherty DD, Bonab AA, Spencer TJ, et al. Dopamine transporter density in patients with attention deficit hyperactivity disorder. Lancet. 1999;354:2132-2133.

33. Scheres A, Milham MP, Knutson B, Castellanos FX. Ventral striatal hyporesponsiveness during reward anticipation in attention-deficit/hyperactivity disorder. Biol Psychiatry. 2007;61:720-724.

34. Rubia K. "Cool" inferior frontostriatal dysfunction in attention-deficit/hyperactivity disorder versus "hot" ventromedial orbitofrontal-limbic dysfunction in conduct disorder: a review. Biol Psychiatry. 2011;69:e69-e87.

35. Castellanos FX, Lee PP, Sharp W, et al. Developmental trajectories of brain volume abnormalities in children and adolescents with attention-deficit/hyperactivity disorder. JAMA. 2002;288:1740-1748.

36. Sowell ER, Thompson PM, Welcome SE, et al. Cortical abnormalities in children and adolescents with attention-deficit hyperactivity disorder. Lancet. 2003;362:1699-1707.

37. Rubia K, Smith AB, Brammer MJ, Toone B, Taylor E. Abnormal brain activation during inhibition and error detection in medication-naive adolescents with ADHD. Am J Psychiatry. 2005;162:1067-1075.

38. Davidson RJ, Jackson DC, Kalin NH. Emotion, plasticity, context, and regulation: perspectives from affective neuroscience. Psychol Bull. 2000;126:890-909. 
39. Davidson RJ, Putnam KM, Larson CL. Dysfunction in the neural circuitry of emotion regulation--a possible prelude to violence. Science. 2000;289:591-594.

40. Posner MI, Rothbart MK. Attention, self-regulation and consciousness. Philos Trans R Soc Lond B Biol Sci. 1998;353:1915-1927.

41. Plessen KJ, Bansal R, Zhu H, et al. Hippocampus and amygdala morphology in attention-deficit/hyperactivity disorder. Arch Gen Psychiatry. 2006;63:795-807.

42. Highfield DA, Hu D, Amsel A. Alleviation of x-irradiation-based deficit in memory-based learning by D-amphetamine: suggestions for attention deficit-hyperactivity disorder. Proc Natl Acad Sci U S A. 1998;95:5785-5788.

43. Duncan RS, Hwang SY, Koulen P. Effects of Vesl/Homer proteins on intracellular signaling. Exp Biol Med (Maywood). 2005;230:527-535.

44. Naisbitt S, Kim E, Tu JC, et al. Shank, a novel family of postsynaptic density proteins that binds to the NMDA receptor/PSD-95/GKAP complex and cortactin. Neuron. 1999;23:569-582.

45. Tu JC, Xiao B, Naisbitt S, et al. Coupling of mGluR/Homer and PSD-95 complexes by the Shank family of postsynaptic density proteins. Neuron. 1999;23:583-592.

46. Zhang GC, Mao LM, Liu XY, et al. In vivo regulation of Homer1a expression in the striatum by cocaine. Mol Pharmacol. 2007;71:1148-1158

47. Yano M, Steiner H. Methylphenidate (Ritalin) induces Homer 1a and zif 268 expression in specific corticostriatal circuits. Neuroscience. 2005;132:855-865

48. de Bartolomeis A, Tomasetti C. Calcium-dependent networks in dopamine-glutamate interaction: the role of postsynaptic scaffolding proteins. Mol Neurobiol. 2012;46:275-296.

49. Kingston AE, O'Neill MJ, Bond A, et al. Neuroprotective actions of novel and potent ligands of group I and group II metabotropic glutamate receptors. Ann N Y Acad Sci. 1999;890:438-449.

50. Hannan AJ, Blakemore C, Katsnelson A, et al. PLC-beta1, activated via mGluRs, mediates activity-dependent differentiation in cerebral cortex. Nat Neurosci. 2001;4:282-288.

51. Wu J, Rush A, Rowan MJ, Anwyl R. NMDA receptor- and metabotropic glutamate receptor-dependent synaptic plasticity induced by high frequency stimulation in the rat dentate gyrus in vitro. J Physiol. 2001;533:745-755.

52. Awad H, Hubert GW, Smith Y, Levey AI, Conn PJ. Activation of metabotropic glutamate receptor 5 has direct excitatory effects and potentiates NMDA receptor currents in neurons of the subthalamic nucleus. J Neurosci. 2000;20:7871-7879.

53. Marino MJ, Wittmann M, Bradley SR, et al. Activation of group I metabotropic glutamate receptors produces a direct excitation and disinhibition of GABAergic projection neurons in the substantia nigra pars reticulata. J Neurosci. 2001;21:7001-7012.

54. Chapman AG, Nanan K, Williams M, Meldrum BS. Anticonvulsant activity of two metabotropic glutamate group I antagonists selective for the mGlu5 receptor: 2-methyl-6-(phenylethynyl)-pyridine (MPEP), and (E)-6-methyl-2-styryl-pyridine (SIB 1893). Neuropharmacology. 2000;39:1567-1574. 